\title{
Xantoma eruptivo com aspecto histopatológico inusitado simulando granuloma anular - Relato de caso
}

\author{
Eruptive xanthoma with unexpected granuloma annulare-like microscopic \\ appearance - Case report
}

\author{
Patrícia P. S. Fagundes ${ }^{1}$ \\ Paulo Augusto Teixeira Pinto ${ }^{3}$ \\ Neusa Yuriko Sakai Valente ${ }^{5}$
}

\author{
Analice Souza Teixeira Pinto ${ }^{2}$ \\ Antônio José Tebcherani ${ }^{4}$
}

\begin{abstract}
Resumo: Xantoma eruptivo e granuloma anular são doenças dermatológicas com quadros clínicos distintos que, algumas vezes, apresentam semelhanças histopatológicas que podem conduzir a diagnóstico errôneo. Relata-se o caso de paciente do sexo masculino com 34 anos, portador de dislipidemia, com lesões clinicamente características de xantoma eruptivo cujo exame histopatológico foi sugestivo de granuloma anular. No entanto, a revisão da lâmina mostrou tratar-se de xantoma eruptivo. A remissão completa e rápida das lesões após o tratamento da dislipidemia confirmou o diagnóstico de xantoma eruptivo e motivou a pesquisa sobre as semelhanças e diferenças histopatológicas entre essas doenças.
\end{abstract}

Palavras-chave: Biópsia; Granuloma anular; Xantomatose

\begin{abstract}
Eruptive xanthoma with unexpected granuloma annulare-like microscopic appearance - Case report Abstract: Eruptive xanthoma and granuloma annulare are dermatological diseases with different clinical findings that, sometimes, exhibit histopathological similarities with potential for misinterpretation. We report a case of an eruption of yellow-orange papules with erythematous borders in a 34-year-old male with high levels of serum triglycerides and cholesterol. The skin biopsy specimen has diagnosed granuloma annulare. Review of the histologic material revealed eruptive xanthoma. Remission of the eruption after treatment of dyslipidemia confirmed the diagnosis of the eruptive xanthoma and motivated research about the histological similarities and differences between these diseases.
\end{abstract}

Keywords: Biopsy; Granuloma annulare; Xanthomatosis

\section{INTRODUÇÃO}

Xantoma eruptivo (XE) é dermatose devida ao depósito de lípides na pele que se manifesta pelo início abrupto de pápulas amarelo-alaranjadas a vermelho-acastanhadas com halo eritematoso, situadas preferencialmente na superfície de extensão das extremidades, principalmente nos joelhos, cotovelos, glúteos, tórax e abdome. ${ }^{1}$ Associa-se a hipertrigliceridemia e níveis elevados de quilomícrons ou de lipoproteínas de densidade muito baixa. Pode também ser observado em pacientes diabéticos não responsivos a insuli- noterapia. Resolução espontânea tem sido relatada. ${ }^{1}$ Por sua vez, o granuloma anular (GA) é dermatose benigna de causa desconhecida, caracterizada em geral por pápula única ou pápulas múltiplas, normocrômicas ou róseas com configuração por vezes anular, preferentemente localizadas no dorso de dedos, mãos e pés. ${ }^{2}$

Histopatologicamente, os achados mais utilizados para diagnóstico são: no XE, aglomerado de macrófagos repletos de lípides sem infiltrado linfocitá-

Recebido em 17.06.2008.

Aprovado pelo Conselho Consultivo e aceito para publicação em 08.11.08

* Trabalho realizado no Serviço de Dermatologia do Complexo Hospitalar Padre Bento - Guarulhos (SP), Brasil.

Conflito de interesse: Nenhum / Conflict of interest: None

Suporte financeiro: Nenhum / Financial funding: None

Médica especialista pela Sociedade Brasileira de Dermatologia - São Paulo (SP), Brasil.

Médica especialista pela Sociedade Brasileira de Dermatologia - São Paulo (SP), Brasil.

Médico assistente do Serviço de Dermatologia do Complexo Hospitalar Padre Bento - Guarulhos (SP), Brasil

Mestre em Medicina, médico patologista do Serviço de Dermatologia do Complexo Hospitalar Padre Bento - Guarulhos (SP), Brasil.

Doutora pelo Departamento de Dermatologia da Faculdade de Medicina da Universidade de São Paulo (USP) - São Paulo (SP), Brasil. 
rio importante ${ }^{1}$ e no GA, reação granulomatosa dérmica multifocal com histiócitos epitelioides e células gigantes multinucleadas, ao redor e entre fibras de colágeno desarranjadas, espessadas e entremeadas por mucina. ${ }^{2}$

\section{RELATO DE CASO}

Doente do sexo masculino, 34 anos de idade, com lesões amareladas no corpo há cerca de 25 dias, assintomáticas. Evoluiu com aumento do número das lesões. Portador de diabetes mellitus tipo 2, em uso de glimepirida e metformina. Negava antecedente familiar de dislipidemias e diabetes mellitus. Ao exame dermatológico, apresentava pápulas e nódulos amarelados e rodeados por halo eritematoso, isolados, localizados no abdome, dorso, glúteos e coxas (Figura 1). Os exames laboratoriais evidenciaram hemograma normal, glicemia de jejum de $210 \mathrm{mg} / \mathrm{dl}$, triglicérides de $2.400 \mathrm{mg} / \mathrm{dl}$, colesterol total de 772 $\mathrm{mg} / \mathrm{dl}$ e soro lipêmico. Os valores de triglicérides superiores a $400 \mathrm{mg} / \mathrm{dl}$ impediram o cálculo das frações. Realizou-se biópsia excisional de pápula amarelada cujo diagnóstico anatomopatológico inicial foi granuloma anular. Foi solicitada revisão de lâmina mediante quadro clínico muito sugestivo de XE e, após análise criteriosa, estabeleceu-se o diagnóstico de xantoma eruptivo, observando-se na coloração pela HE, na derme superior, a presença de infiltrado nodular com histiócitos, alguns dos quais com citoplasma espumoso (Figuras 2 e 3). Coleções menores desses histiócitos se dispunham ao redor de vasos. À coloração pelo alcian-blue/PAS, mucina foi observada em meio aos histiócitos e às fibras colágenas desarran-



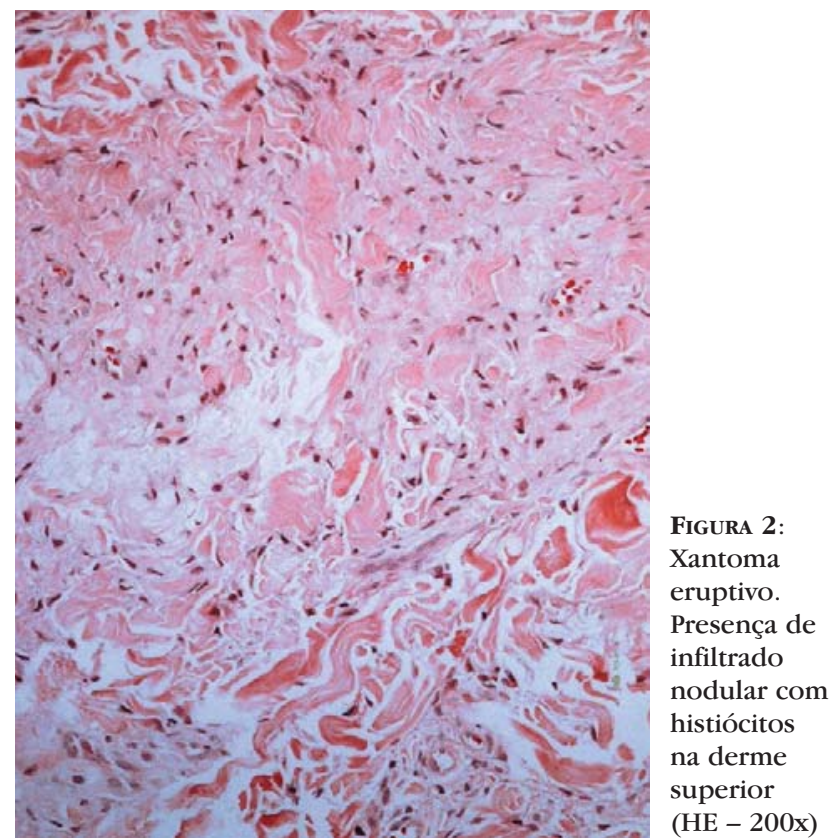

jadas (Figura 4). À coloração de Weigert, havia rarefação de fibras elásticas nas áreas ocupadas pelo infiltrado histiocitário (Figura 5).

O paciente foi encaminhado para tratamento da dislipidemia e após 15 dias da introdução de dietoterapia e bezafibrato apresentou involução completa das lesões (Figura 6). Essa resposta clínico-dermatológica seria pouco provável se o paciente apresentasse granuloma anular disseminado, que, apesar de ter involução espontânea descrita, apresenta mais comumente persistência prolongada, até mesmo por décadas. ${ }^{3} \mathrm{O}$ tratamento para o GA disseminado pode ser tentado com antimaláricos, corticóides, drogas citotóxicas, niacinamida, PUVA, etretinato, isotretinoína, vitamina $\mathrm{E}$, inibidores da 5-lipoxigenase, tacrolimus, pimecrolimus e infleximab, todos com respostas variáveis. ${ }^{4-10}$ Não há, até $\mathrm{o}$ momento da conclusão deste artigo, relato na literatura médica de granuloma anular disseminado que houvesse regredido com a utilização de bezafibrato.

\section{DISCUSSÃO}

Xantoma eruptivo e granuloma anular são dermatoses clinicamente distintas que, no entanto, podem apresentar características histológicas em comum. Esse fato tem sido pouco relatado na literatura. ${ }^{11}$

Cooper et al. compararam a presença dos seguintes aspectos histopatológicos no XE e GA: ${ }^{11}$ 1- uma ou múltiplas lesões na derme superficial; 2infiltrado intersticial e perivascular; 3- desarranjo da derme reticular; 4- histiócitos intersticiais; 5- histiócitos perivasculares; 6- linfócitos perivasculares; 7- linfócitos intersticiais; 8- histiócitos xantomizados; 9- his- 


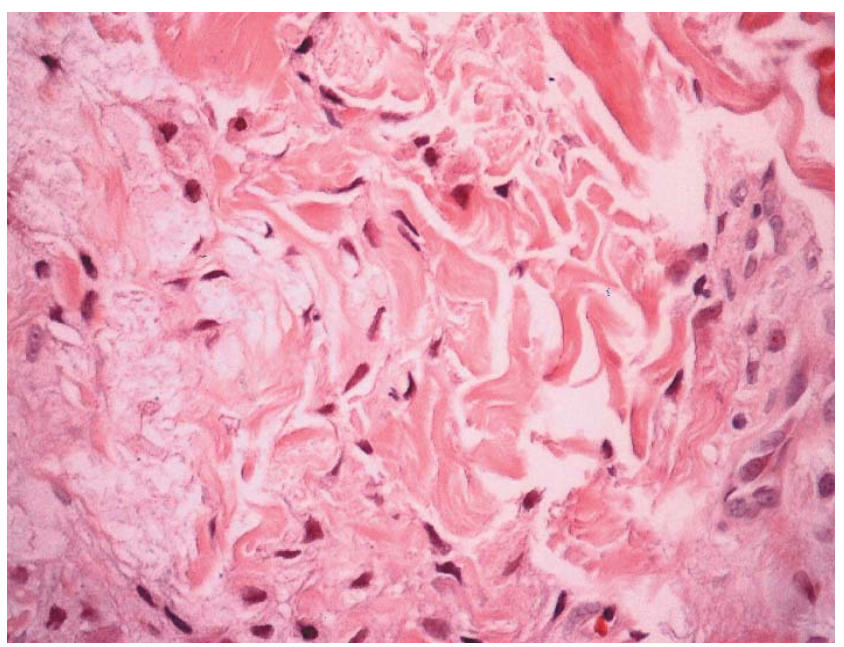

Figura 3: Xantoma eruptivo. Infiltrado dérmico com histiócitos de citoplasma por vezes espumoso (HE - 400x)



Figura 4: Xantoma eruptivo. Mucina permeando os histiócitos e fibras colágenas (alcian-blue/ PAS - 100x)

tiócitos epitelioides; 10- células multinucleadas; 11depósitos de lipídios; 12- necrobiose; 13- necrose celular; 14- depósito de ácido hialurônico; 15- alteração das fibras elásticas.

Os quatro primeiros itens listados acima foram observados em ambas as doenças, indistintamente. Para os demais aspectos analisados, houve diferenças entre as doenças: os histiócitos perivasculares e os histiócitos xantomizados são numerosos no XE e em número reduzido ou ausentes no GA; depósitos de lipídios estão presentes no XE e ausentes no GA; os linfócitos perivasculares e intersticiais, histiócitos epitelioides e células multinucleadas estão em número reduzido ou ausentes no XE e numerosos no GA; necrobiose e evidência de necrose celular estão



Figura 5: Xantoma eruptivo. Rarefação de fibras elásticas na área do infiltrado histiocitário dérmico (Weigert - 100x)

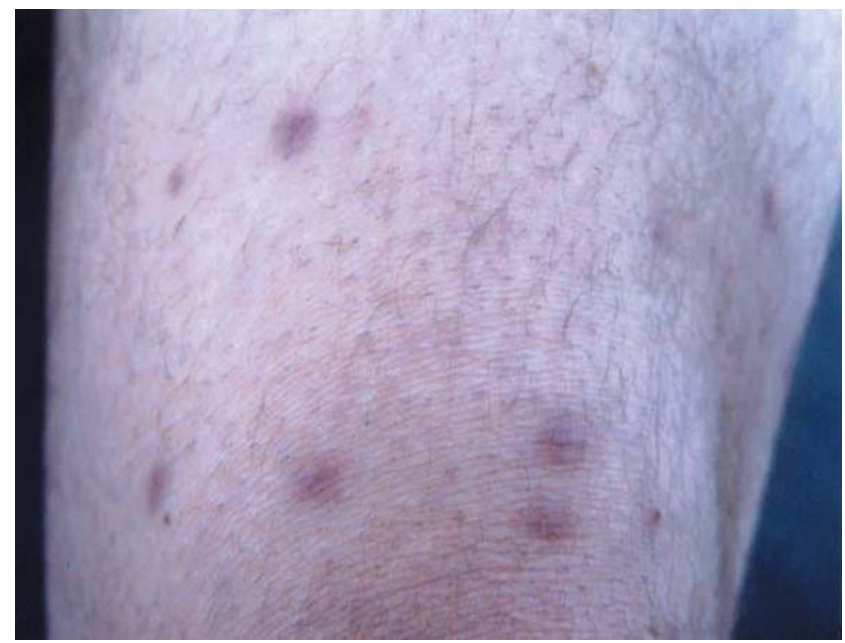

Figura 6: Involução das lesões 15 dias após dietoterapia e bezafibrato

ausentes no XE e presentes no GA; depósito de ácido hialurônico pode ser visto em ambas as doenças, porém no XE situa-se na borda e, no GA, no centro das lesões; as fibras elásticas não estão claramente afetadas no XE e apresentam-se reduzidas ou ausentes quando se observa uma necrobiose bem estabelecida no GA. Entretanto, há que se dizer que a coloração para fibra elástica pode não revelar alterações caso a lesão seja recente.

Ao se aplicarem os critérios acima listados, na revisão da lâmina deste caso, as características histopatológicas encontradas que permitiram o diagnóstico de XE foram: presença de histiócitos xantomatosos e ausência de células gigantes multinucleadas e de infiltrado linfocitário associado.

Limitando-se a observação apenas à presença de mucina e à rarefação das fibras elásticas vistas nas figuras 5 e 6 , respectivamente, poder-se-ia de fato 
sugerir o diagnóstico de GA. Talvez tenha sido o ocorrido quando do primeiro laudo histopatológico.

Importante ressaltar que a pesquisa para gordura não foi realizada devido à impossibilidade de se repetir a biópsia pela pronta resposta do paciente ao tratamento.

Segundo Ackerman, ${ }^{12}$ a diferença crucial entre XE e GA é o depósito de lípides extracelulares no centro da paliçada de histiócitos no XE, em contraste com o depósito de mucina no GA.

Como visto no presente caso relatado, é possível chegar a um diagnóstico histopatológico precipita- do e errôneo se apenas algumas características forem analisadas. Além de Cooper et al., esse fato já havia sido descrito por Ackerman e Pistone. ${ }^{12-13}$

O objetivo do relato deste caso é ressaltar a existência de semelhanças histopatológicas entre essas duas doenças clinicamente tão distintas e, em decorrência disso, assinalar a importância de se valorizarem suas apresentações clínicas.

A semelhança histopatológica possível entre XE e GA, desconhecida por muitos patologistas devido à escassez de relatos na literatura, pode explicar o diagnóstico histopatológico inicial errôneo do caso em foco.

\title{
REFERÊNCIAS
}

1. Seymour CA. Xantoma and abnormalities of lipid metabolism and storage. In: Burns T, Breathnach S, Cox N, Griffiths C. Rook's Textbook of Dermatology. 7th ed. Oxford: Blackwell Science; 2004. p. 57.65-8.

2. Burns DA. Granuloma annulare. In: Burns T, Breathnach S, Cox N, Griffiths C. Rook's Textbook of Dermatology. 7th ed. Oxford: Blackwell Science; 2004. p. 57109-119.

3. Ma A, Medenica M. Response of generalized granuloma annulare to high-dose niacinamide. Arch Dermatol. 1983;119:836-9.

4. Sahin MT, Ermertcan AT, Ozturkcan S, Turkdogan P. Generalized granuloma annulare in a patient with type II diabetes mellitus: successful treatment with isotretinoin. J Eur Acad Dermatol Venereol.. 2006;20:111-3.

5. Asano Y, Saito A, Idezuki T, Igarashi A. Generalized granuloma annulare treated with short-term administration of etretinate. J Am Acad Dermatol. 2006;54:S245-7.

6. Smith KJ, Norwood C, Skelton H. Treatment of disseminated granuloma annulare with a 5-lipoxygenase inhibitor and vitamin E. Br J Dematol. 2002;146:667-70.

7. Setterfield J, Huilgol SC, Black MM. Generalised granuloma annulare successfully treated with PUVA. Clin Exp Dermatol. 1999;24:458-60.

8. Harth W, Linse R. Topical tacrolimus in granuloma annulare and necrobiosis lipoidica. Br J Dermatol. 2004; 150:792-4.

9. Rigopoulos D, Prantsidis A, Christofidou E, Ioannides D, Gregoriou S, Katsambas A. Pimecrolimus 1\% cream in the treatment of disseminated granuloma annulare. Br J Dermatol. 2005;152:1364-5.

10. Hertl MS, Haendle I, Schuler G, Hertl M. Rapid improvement of recalcitrant disseminated granuloma annulare upon treatment with the tumour necrosis factor-alpha inhibitor, infliximab. $\mathrm{Br} \mathrm{J}$ Dermatol. 2005; 152:552-5.

11. Cooper PH. Eruptive xanthoma: a microscopic simulant of granuloma annulare. J Cutan Pathol. 1986;13:207-15

12. Pistone GA. Pathology quiz case 1: eruptive xanthoma (associated with type 5 hyperlipoproteinemia). Arch Dermatol. 1984;120:1366-8.

13. Ackerman AB. Histologic diagnosis of inflammatory skin diseases. Philadelphia: Lea \& Febiger; 1978.

\author{
ENDEREÇO PARA CORRESPONDÊNCIA / MAILING ADDRESS: \\ Patrícia P. S. Fagundes \\ Rua Jacques Félix, 96 110R V. N. Conceição \\ 04509-000 São Paulo SP \\ Tel./fax: (11)38426208 \\ patriciasfagundes@yaboo.com.br
}

Como citar este artigo/How to cite this article: Fagundes PPS, Pinto AST, Pinto PAT, Valente NYS, Tebcherani AJ. Xantoma eruptivo com aspecto histopatológico inusitado simulando granuloma anular - Relato de caso. An Bras Dermatol. 2009;84(3):289-92. 